CARNETS DE Carnets de géographes

GÉOGRAPHES.

$1 \mid 2010$

Varia

\title{
Environnement et littoralisation de l'ouest algérien
}

\section{Tarik Ghodbani}

\section{OpenEdition}

Journals

Édition électronique

URL : http://journals.openedition.org/cdg/2105

DOI : $10.4000 /$ cdg. 2105

ISSN : 2107-7266

\section{Éditeur}

UMR 245 - CESSMA

\section{Référence électronique}

Tarik Ghodbani, «Environnement et littoralisation de l'ouest algérien », Carnets de géographes [En ligne] 1 | 2010, mis en ligne le 01 octobre 2010, consulté le 24 septembre 2020. URL : http:// journals.openedition.org/cdg/2105; DOI : https://doi.org/10.4000/cdg.2105

Ce document a été généré automatiquement le 24 septembre 2020

\section{(c) (i) () $९$}

La revue Carnets de géographes est mise à disposition selon les termes de la Licence Creative Commons Attribution - Pas d'Utilisation Commerciale - Pas de Modification 4.0 International. 


\title{
Environnement et littoralisation de l'ouest algérien
}

\author{
Tarik Ghodbani
}

1 Thèse relevant de la géographie de l'environnement, ce travail combine les connaissances de la géographie physique et de la géographie humaine. L'aménagement de l'espace littoral algérien et les questions environnementales sont les axes centraux de cette thèse. Le terrain d'étude est le littoral Nord - Ouest de l'Algérie. Il sert de test à nos méthodes de travail et c'est un cas d'école pour la diversité de son occupation et de son exploitation par l'homme. Cet espace regroupe des villes importantes avec à leur tête Oran, deuxième ville d'Algérie, qui compte plus d'un million d'habitants. Il polarise un arrière-pays étendu et englobe des zones industrielles d'envergure, des infrastructures portuaires parmi les plus importantes du pays ainsi que quelques stations touristiques balnéaires. L'urbanisation et le développement de ces activités économiques se sont accélérés ces dernières décennies et ont eu des impacts qui représentent actuellement des facteurs de risque qu'il faut gérer et maîtriser. La multiplicité des formes de dégradation de l'environnement nous renseigne non seulement sur le poids de l'action anthropique mais aussi sur la fragilité des écosystèmes composant le littoral.

2 L'objectif de l'étude est d'analyser les relations homme/milieu dans un espace convoité et fragile. Littoralisation et dégradation du milieu naturel en sont les conséquences visibles. La dégradation de l'environnement apparaît par une dynamique perturbée, une biodiversité en régression et un paysage naturel artificialisé. Le choix de travailler sur le littoral Ouest s'est avéré à la fois difficile et pertinent. La pertinence est relative à deux éléments : le premier est la rareté des études de ce type sur cet espace. Le second élément et la variété des occupations humaines et économiques et la richesse paysagère que représente ce littoral. Pour plus d'efficacité dans l'approche adoptée, deux méthodes ont été utilisées: la diachronie, et le multiscalaire. La diachronie permet d'étudier l'évolution dans le temps des interactions entre occupation du littoral et milieu naturel. Le multiscalaire permet d'analyser les différents phénomènes en passant par plusieurs niveaux d'échelles, du local au régional, et inversement. L'étude 
était basée sur neuf cas d'études. Le critère de choix est essentiellement lié à la nature de l'action anthropique (aménagement portuaire, urbanisation, prélèvement, artificialisation) et le type de l'espace naturel (embouchure, marais, île, dune plage...).

Pour chaque cas, une ou plusieurs problématiques ont été posées, elles sont insérées dans le texte selon des entrées thématiques qu'on a organisées en trois grandes parties : la première sur l'état de l'environnement, la deuxième sur la littoralisation, comme élément explicatif à la dégradation de l'environnement et la troisième sur le jeu d'acteurs, les outils de gestion et les conflits d'usage.

La fragilité des écosystèmes dans le littoral Ouest de l'Algérie se manifeste à travers l'urbanisation et l'artificialisation de la côte, l'exploitation intensive des ressources naturelles comme le sable dunaire, l'eau ou le stock halieutique...etc. À titre d'exemple, on peut citer le cas de Terga. À Terga les déséquilibres environnementaux représentent des facteurs de risques. La zone côtière de Terga englobe un espace dunaire exploité actuellement par une grande sablière qui alimente presque tous les chantiers de la région ouest. Les extractions massives pratiquées sur cette dune, et qui dépassent les limites prescrites par la loi minière algérienne, ont conduit à l'inondation de l'oued El Malleh le 13 octobre 2000, suite à des pluies torrentielles. La disparition de la partie dunaire faisant barrière au débordement de l'oued sur sa rive gauche a engendré l'inondation de la partie nord de la plage, la destruction de 50 cabanons et la mort d'un jeune garçon. À ces perturbations d'ordre dynamique et fluvial, s'ajoutent d'autres impacts négatifs: l'affleurement de la nappe alluviale, l'ensablement des terres agricoles proches de la dune et enfin la dégradation de la qualité paysagère du site naturel. L'action anthropique est l'élément perturbateur des écosystèmes. Elle est le résultat de la littoralisation qui se manifeste par une concurrence sur l'exploitation des ressources naturelles et sur l'appropriation du foncier littoral pour une éventuelle urbanisation. La répartition de la population dans l'Oranie montre une forte densité dans le nord. $24 \%$ de la population réside dans les communes à façade maritime. Les plus forts taux de croissance sont par ailleurs enregistrés dans les communes périphériques aux zones fortement urbanisés : Oran, Mostaganem, Arzew, Ghazaouet et Béni-Saf. Cette répartition épouse le relief ; les zones de plaines sont les plus densément peuplées. Des zones montagneuses comme l'Ouarsenis ont en revanche un déficit migratoire de $20 \%$.

5 Les conditions naturelles à elles seules ne peuvent expliquer ces écarts. Le renforcement, par la colonisation, d'un réseau de villes littorales et d'un réseau routier transversal est à l'origine de la littoralisation, renforcée après l'indépendance par l'implantation de pôles, de zones industrielles et par la récente ouverture sur l'économie de marché conjuguée à la privatisation. Des mesures de rééquilibrage par les découpages administratifs vers le début des années 80 ont tenté de réduire le poids de la littoralisation. Les efforts se limitent à l'espace tellien, les espaces sahariens et steppiques restant à la marge de ce volontarisme étatique. Plus récemment une autre politique semble se tracer pour une meilleure protection des milieux naturels fragiles, dont le littoral. En effet, un nombre important de textes juridiques sont promulgués depuis 1990.

6 La législation algérienne souhaite donc préserver les milieux naturels sensibles. Cependant l'application de ces instruments reste difficile et l'état de l'environnement littoral ne cesse de se dégrader. Cette difficulté apparait principalement dans l'incompatibilité entre les outils de gestion, la mise à l'écart des acteurs locaux par le 
pouvoir central dans la prise de décision, la vision sectorielle dans les opérations d'aménagement et enfin l'absence de complémentarité entre activités économiques littorales. En plus de ces résultats, la recherche sur la littoralisation et l'environnement de l'Ouest algérien a mis en évidence la nécessité de multiplier les travaux sur l'ensemble du littoral algérien. Les résultats de ces études permettront d'approfondir les connaissances sur les relations homme/milieu dans la cadre d'une future gestion intégrée des zones côtières (GIZC). Les enjeux fonciers, les pratiques, les perceptions et le jeu d'acteurs dans le domaine de la gestion des zones côtières fragiles pourront constituer des thèmes privilégiés. Des comparaisons avec d'autres littoraux seraient d'un grand intérêt.

\section{Fiche informative}

\section{Discipline}

Géographie

\section{Directeurs}

Bachir TIDJANE et Bouziane SEMMOUD

\section{Université}

Université d'Oran Es-Senia

Membres du jury de thèse, soutenue le 28 septembre 2009

- M. Bachir Tidjane, Université d'Oran Es-Senia

- M. Bouziane Semmoud, Université de Paris VIII

- M. Ali Hadjiedj, Université d'Alger

- M. Marc Côte, Université d'Aix Marseille I

- Mme Françoise Plet, Université Paris 8

- M. Mustafa Chachoua, Université d'Oran

Situation professionnelle actuelle

Maître de conférence à l'Université d'Oran.

\section{Contact de l'auteur}

ghodbani_tarik[at]yahoo.fr

\section{INDEX}

Thèmes : Carnets de soutenances 